\title{
1 Inferring ongoing cancer evolution from single tumour biopsies using synthetic supervised learning
}

\author{
Authors: Tom W. Ouellette ${ }^{1,2}$ and Philip Awadalla $a^{1,2^{*}}$
}

\author{
Affiliations: \\ ${ }^{1}$ Ontario Institute for Cancer Research, Department of Computational Biology, Toronto, Ontario M5G 0A3, \\ Canada \\ ${ }^{2}$ Department of Molecular Genetics, Temerty Faculty of Medicine, University of Toronto, Toronto, Ontario \\ M5S 1A8, Canada \\ $11{ }^{*}$ Correspondence to: tom.ouellette@oicr.on.ca or philip.awadalla@oicr.on.ca
} Keywords: cancer evolution, subclonal selection, variant allele frequencies, population genetics, simulation, synthetic data, deep learning, transfer learning

\section{Abstract}

Variant allele frequencies (VAF) encode ongoing evolution and subclonal selection in growing tumours. However, existing methods that utilize VAF information for cancer evolutionary inference are compressive, slow, or incorrectly specify the underlying cancer evolutionary dynamics. Here, we provide a proof-ofprinciple synthetic supervised learning method, TumE, that integrates simulated models of cancer evolution with Bayesian neural networks, to infer ongoing selection in bulk-sequenced single tumour biopsies. Analyses in synthetic and patient tumours show that TumE significantly improves both accuracy and inference time per sample when detecting positive selection, deconvoluting selected subclonal populations, and estimating subclone frequency. Importantly, we show how transfer learning can leverage stored knowledge within TumE models for related evolutionary inference tasks - substantially reducing data and computational time for further model development and providing a library of recyclable deep learning models for the cancer evolution community. This extensible framework provides a foundation and future directions for harnessing progressive computational methods for the benefit of cancer genomics and, in turn, the cancer patient. TumE is publicly available for use at https://github.com/tomouellette/TumE. 


\section{Introduction}

30 Cancer is a disease characterized by unrelenting tissue growth and clonal evolution. During evolution, genetic and epigenetic aberrations provide the reservoir for dysfunctional cellular phenotypes that maintain a tumour's replicative advantage, while, over time, fluctuating physiological and ecological properties within the tumour microenvironment drive the need for updated adaptations that sustain immortality ${ }^{1}$. Overall, the complex interplay between mutation accumulation and microenvironmental changes leads to a high degree of both cellular and genetic heterogeneity and, by proxy, composite subclonal structure in tumours ${ }^{2-4}$. Naturally, the desire to better understand the evolutionary and subclonal dynamics in growing tumour populations has become a major task for cancer genomics - with goals of forecasting tumour progression, developing adaptive evolutionary therapies, and deconvoluting the genetic architecture that drives adaptation $^{3,5-8}$.

However, a significant hurdle in understanding cancer evolution in vivo are the clinical constraints surrounding serial sequencing, through space or time. For this reason, tumour biopsies are primarily sequenced in bulk from a single site and at a single time point. Although multi-region and single-cell data are becoming increasingly utilized, single time point, bulk sequenced biopsies still represent the major accessible data source for precision genomics guided treatment ${ }^{9}$ and for studying cancer genomics and evolution in patients ${ }^{10,11}$. Given this limitation, a reasonable strategy for inferring evolution in single tumor biopsies has been to utilize theoretical population genetics to capture signatures of selection from the variant allele frequency (VAF) distribution ${ }^{7,12-17}$. The premise being that fitness-altering mutations will deterministically change in frequency over time, leading to characteristic and quantifiable deviations in the VAF distribution relative to some neutral evolutionary scenario ${ }^{18}$.

VAF-based methods have been employed to differentiate between positive selection and neutral evolution ${ }^{12,13}$, to examine growth patterns ${ }^{19}$, to quantify subclonal fitness and time subclonal emergence ${ }^{7,15}$, and to build population genetics informed mixture models ${ }^{16}$ that account for neutral dynamics, that shape, to some extent, all tumour populations. With that said, existing VAF-based methods used to infer cancer evolution, although mechanistic and useful, have apparent limitations. For example, single statistics ${ }^{12,20,21}$ are maximally compressive and cannot infer complex information, approximate Bayesian computation methods suffer from the curse of dimensionality and can be prohibitively slow due to a rate-limiting simulation step required for each sample ${ }^{7,22,23}$, and mixture models, used to identify subclonal populations ${ }^{16,24,25}$, are only implicitly connected to an underlying model of evolution and, until recently ${ }^{16}$, have been built under incorrect assumptions that have led to systematic overestimation in the number of subclonal populations in sequenced tumours.

64 To address these limitations, we contribute a proof-of-principle synthetic supervised deep learning 65 approach, TumE, for quantifying and classifying the evolutionary and subclonal dynamics in bulk sequenced 
tumours biopsies using purity-corrected variant allele frequency (VAF) information from diploid genomic regions. By generating synthetic VAF distributions, as a proxy for evolutionary ground truth, from plausible simulations of tumour evolution, we were able to build inference models that accurately classify and quantify evolutionary (e.g. positive selection versus neutral evolution) and subclonal dynamics (e.g. subclone frequency) in real patient tumours while capturing uncertainty in our estimates, via a form of approximate Bayesian inference called Monte Carlo dropout ${ }^{26,27}$. Importantly, our method further highlights the power of utilizing deep learning for inference - namely exploiting stored knowledge via transfer learning. By recycling our models for new evolutionary prediction tasks, we reduce the computational burden associated with the generation of synthetic or simulated data. We validated our synthetic supervised learning approach in millions of synthetic tumours and applied TumE to 95 copy-number and purity corrected whole-genome (WGS) and whole-exome (WES) sequenced tumour biopsies.

\section{Inferring cancer evolution using synthetic supervised deep learning} approximate inference under a generative mode ${ }^{28}$. Therefore, by optimizing a neural network using realistic synthetic data $\mathbf{x}$ generated from a stochastic generative process $p(\mathbf{x}, \mathbf{z} \mid \boldsymbol{\theta})$, where $\boldsymbol{\theta}$ indicates the prior or parameters that define the simulation and $\mathbf{z}$ indicates the latent variables generated during simulation, we can build inference models that approximate our true posterior of interest $p(\boldsymbol{\theta}, \mathbf{z} \mid \mathbf{x})$. In our case, by optimizing a neural network using synthetic VAF distributions sampled from $p(\mathbf{x}, \mathbf{z} \mid \boldsymbol{\theta})$, we can build inference models for evolutionary inference in sequenced tumour biopsies (Figure 1a-c; Methods).

To generate synthetic data that properly captured evolutionary dynamics in patient tumours, we implemented a simulation framework, i.e. a stochastic generative process $p(\mathbf{x}, \mathbf{z} \mid \boldsymbol{\theta})$, combining two complementary approaches to improve the speed and efficiency of synthetic data generation - one for tumours subject to positive selection and one for tumours evolving neutrally (Figure 1a).

For growing tumours simulated with positive selection, we utilized a well-established framework of cancer evolution that models exponential tumour growth under a stochastic branching process ${ }^{7,12,13,15,19,30}$ and coupled this with a virtual biopsy procedure to account for sequencing noise observed in real patient tumours (adapted from ref $^{7}$ ). In our model, we allowed for a completely stochastic arrival of driver mutations that multiplicatively increased the fitness of mutated subclones, and tracked the frequency of each subclone until the time of virtual biopsy (Methods). In this study, we define a subclone as a subpopulation of cells with a fitness or growth rate advantage relative to the background population (Methods) and consider subclones detectable if they are between $\sim 10-40 \%$ VAF (20 - 80\% cellular fraction). 

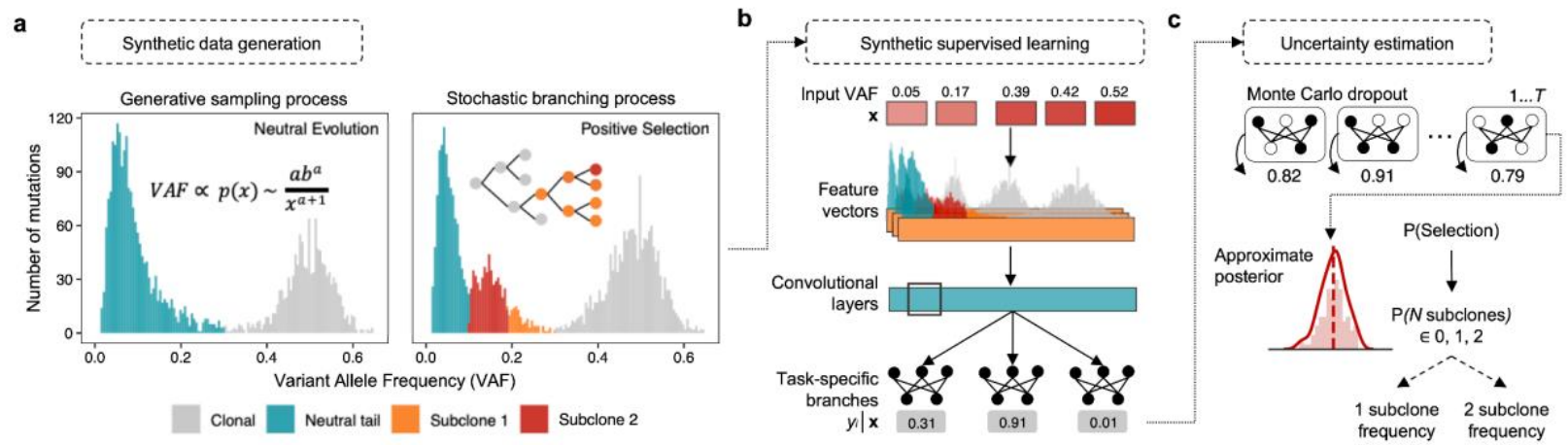

Figure 1. (a) TumE integrates a generative sampling process and stochastic simulation of cancer evolution to build well-specified synthetic variant allele frequency (VAF) distributions with respect to data observed in bulk sequenced tumour biopsies. Assuming copy-neutral diploid regions of tumour genomes, the generative sampling process uses the observation that neutral VAF distributions can be described by a power-law or Pareto neutral 'tail' ${ }^{16,29}$ in addition to a dispersed clonal peak. By sampling empirically valid Pareto distributions, rapid realizations of the null hypothesis of neutral evolution encoded in the VAF distribution can be created (Methods). The stochastic branching process model of tumour evolution is then used to link parameters and latent states, relevant to positive subclonal selection, back to VAF distributions (Methods). (b) Synthetic supervised learning utilizes neural networks capable of handling the complete dimensionality of the simulated VAF distributions, $\mathbf{x}$, to solve the inverse problem of identifying the evolutionary parameters and latent states, $\mathbf{y}$, assigned to each synthetic VAF distribution. (c) We can then quantify model uncertainty using a computationally efficient form of Bayesian deep learning called Monte Carlo dropout ${ }^{26,27}$. Approximate posteriors are generated by performing $T$ stochastic passes through the trained neural network.

For tumours that lacked selected subclones (neutrally evolving), we implemented a generative sampling process based on the observation that VAF distributions from tumours without positively selected subclones can be described by a power-law or Pareto distribution ${ }^{16,29}$ in conjunction with a dispersed clonal peak (Supplementary Figure 1). Concisely, this process involved i) sampling allele frequencies from empirically realistic Pareto distributions to generate the neutral power-law 'tail' in the VAF distribution, ii) adding additional diploid clonal heterozygous mutations at 50\% VAF, and then iii) injecting additional sequencing noise under a beta-binomial model (Methods). In general, a complete VAF distribution indicative of positive selection, and computed from heterozygous diploid mutations, includes a neutral power-law tail ${ }^{12,16}$, a heterozygous clonal peak centered at $\sim 50 \% \mathrm{VAF}$, and additional subclonal peak(s) in the intermediate frequency ranges ( 10 - 40\% VAF); whereas a neutrally evolving tumour, or one with undetectable selected subpopulations, lacks the characteristic subclonal peak(s) (Figure 1a). To ensure positively selected and neutrally evolving synthetic tumours were not out of distribution with each other given the alternate data generation approaches, we simulated synthetic tumours in pairs, assigning the neutral VAF distributions with equivalent parameters and mutations with respect to the paired positive selection simulation (Methods; pseudo algorithms and examples provided alongside Supplementary Figure $2 \& 3$ ).

Using this framework, we generated approximately 40 million synthetic tumours across varying mutation rates, selection coefficients, and sequencing noise parameters. We selected broad simulation parameter ranges that were consistent with previous computational studies and empirically estimated values (Methods; Supplementary Table S1). By generating synthetic tumours using well-specified simulations (comparison of real and synthetic data outlined in Methods and Supplementary Figures 3 - 5), we were able 
subclonal and evolutionary dynamics. We then used the millions of annotated synthetic VAF distributions to train hundreds of neural networks using a random hyperparameter search to make inferences on the evolutionary mode (positive selection or neutral evolution), the number of subclones $(0,1$, or 2 ), and the subclone frequency at borderline to optimal sequencing depths (50 - 250X) for evolutionary analysis in cancer genomics (Figure 1b; Methods). To capture model-based uncertainty in our estimates, we implemented a form of Bayesian approximation for deep learning called Monte Carlo (MC) dropout ${ }^{26,27}$ (Figure 1c; Methods). We used MC dropout to mitigate overconfident estimates in cases of high uncertainty or broad approximate posteriors. In general, we structured both neural network training and prediction to favour the more parsimonious explanation of the data (fewer subclones and neutral evolution; Methods). We show how using a classification threshold based on a lower bound of the MC dropout approximate posterior helps mitigate model overconfidence across changing subclone mutational burdens and frequencies in Supplementary Figure 6. Following training, we selected the top scoring models, for predicting the evolutionary mode, number of subclones, and subclone frequency, for further validation (Methods).

We outline the full synthetic supervised learning pipeline in Methods. In addition, we highlight that even though we model VAF distributions in patient tumours using point mutations from diploid regions, mutations in our framework, as with previous approaches ${ }^{7,13,16}$, are agnostic to the underlying functional alteration, e.g. missense, silent, driver or copy number driving selection in patient tumours. This is because genomewide linkage, a by-product of zero recombination, results in hitchhiking of any additional point mutations on the genetic background of any selected clone ${ }^{3,18}$.

\section{Comparison of synthetic supervised learning to existing methods}

To evaluate TumE performance on inferred estimates of selection, number of subclones, and subclone frequency, we simulated an additional $~ 2.8$ million synthetic tumours under neutral evolution ( 0 subclones) and positive selection ( 1 or 2 detectable selected subclones) assessing the impact of variable sequencing depths (50 - 250x coverage) and read count overdispersion (0 - 0.3 rho) (Methods). We first compared TumE against frequency-based summary statistic approaches for differentiating between neutral evolution and positive selection and found that TumE significantly outperforms recently developed VAF summary statistics $^{12}$ (two-sided Wilcoxon test, $p=2.7 \times 10^{-12}$ ) as well as common population genetic summary statistics $^{20,21}$ (two-sided Wilcoxon test, $p=1.9 \times 10^{-8}$ ), based on AUROC (Figure 2a). Further, TumE outperforms each statistic individually when compared across all sequencing depth and overdispersion combinations analyzed here (ROC analysis; Supplementary Figure 7).

We next compared TumE against the only mixture model approach, MOBSTER ${ }^{16}$, that explicitly and correctly considers the neutral dynamics within sequenced tumour VAF distributions to detect subclones. 
(precision-recall, Supplementary Figure 8 ) and for predicting subclone frequency (Figure 2b; correlation and mean absolute percentage error, Supplementary Figure 9) across all empirically relevant depth (50 $250 x$ coverage) and read count overdispersions ( 0 - 0.003 rho) combinations. However, as expected, we found that the performance of TumE and MOBSTER both degrade as sequencing depth decreases $(\leq 75 x$ coverage) and overdispersion increases $(\geq 0.01)$ under a beta-binomial sequencing noise model (Supplementary Figure 8 \& 9). Furthermore, additional analysis of subclone frequency estimates in the 2 subclone setting revealed that as inter-subclone distance increases, i.e., overlap of subclonal peaks decrease, the mean percentage error for predicting the frequency of both the lowest and highest frequency subclone decreases towards zero (Supplementary Figures 10 - 12).

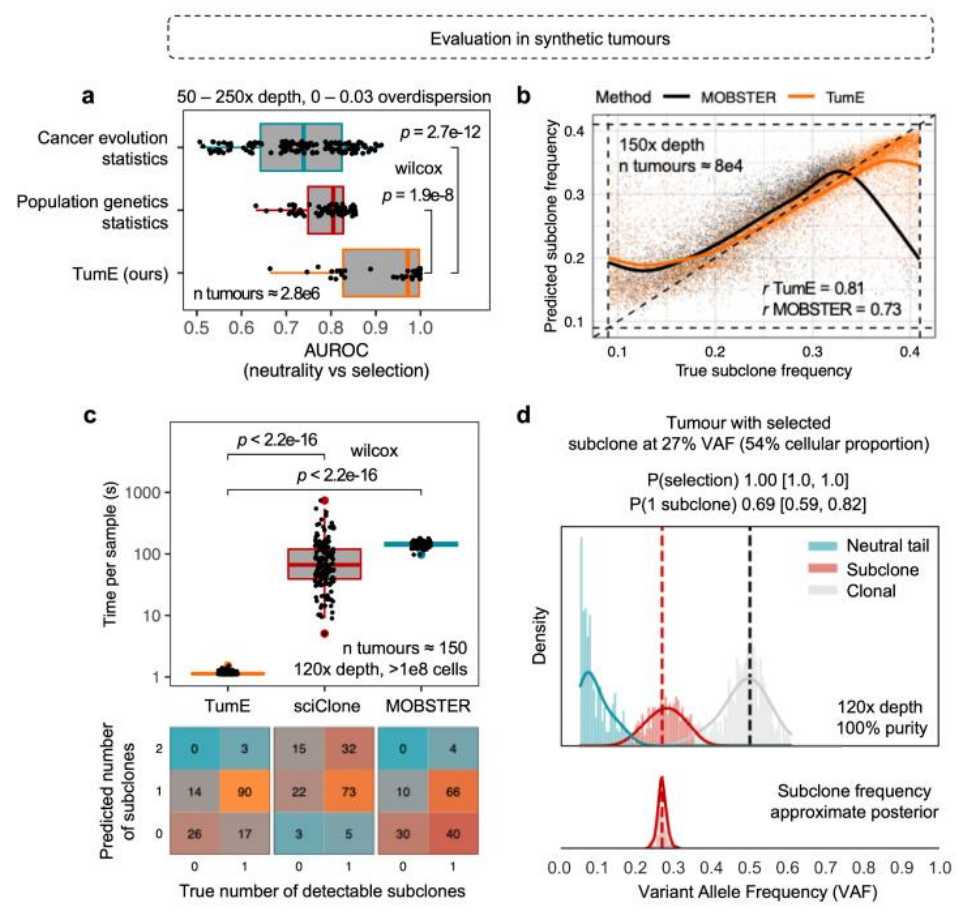

(a) In a cohort of 2.8 million synthetic tumours, TumE outperformed all existing common population genetic ${ }^{20,21}$ and cancer evolution ${ }^{7,12}$ specific summary statistics when differentiating between positive selection and neutral evolution, based on AUROC (two-sided Wilcoxon test). (b) Further, for predicting the true frequency of selected subclones, TumE provides comparable or better performance relative to the current state-of-the-art mixture model MOBSTER ${ }^{16}$ that properly accounts for neutral dynamics in tumour populations. The panel shows correlation between the true and predicted subclone frequency in 80,000 synthetic tumours sequenced at $150 x$ mean sequencing depth. (c) In an orthogonal dataset of 150 synthetic tumours ${ }^{16}$ with either 0 or 1 detectable subclones, TumE was significantly faster at estimating the number of subclones (two-sided Wilcoxon test) than existing mixture model based methods sciClone $^{24}$ and MOBSTER ${ }^{16}$ (measured in inference time per sample). In addition, only TumE and MOBSTER consistently identified the correct number of subclones, as both methods directly account for the neutral dynamics observed in tumour populations. (d) TumE estimates in a synthetic tumour sequenced at $120 x$ mean sequencing depth and a subclone at $54 \%$ cellular fraction.

171 Given our simulation framework was based on certain approximating assumptions to improve 172 computational speed and efficiency (namely small population size and no cell death; outlined in Methods), 173 we sought to perform additional validation of evolutionary estimates in an alternative dataset of synthetic 174 tumours $^{16}$. The orthogonal dataset, described in Caravagna et al. $2020^{16}$, consisted of 150 synthetic 175 tumours, 40 effectively neutral and 110 with one detectable subclone (between 10 - 45\% VAF), sequenced 
to $120 x$ depth and grown to a population size of $>10^{8}$ cells at birth rate of 1 and death rate of 0.2 . To frame our predictions relative to existing methods, we applied TUmE, MOBSTER, and a variational Bayesian mixture model sciClone ${ }^{24}$ to the synthetic dataset. To make comparisons fair, we limited the maximum number of subclonal cluster assignments to 2 for both MOBSTER and sciClone, as this was the upper bound on TumE estimates (Methods). For sciClone, this meant setting the maximum number of mixture components to 4 (neutral tail, 2 subclones, and a clonal peak) as sciClone doesn't properly account for neutral dynamics (Pareto tail) observed in sequenced tumour populations. Both TumE and MOBSTER consistently identified the correct number of detectable subclones in the majority of cases while sciClone systematically overestimated the number of subclones, even after correcting estimates for the clonal peak and neutral tail (Figure 2c). However, relative to MOBSTER, which tended to converge to more parsimonious explanations of the data (i.e., 0 subclones), TumE was able to detect subclones at a higher rate (Figure 2c). In addition, compared to both sciClone and MOBSTER, TumE provided orders-ofmagnitude faster estimates (two-sided Wilcoxon test, $p<2.2 \times 10^{-16}$, Figure $2 \mathrm{c}$ ), reducing run times per sample from minutes to $\sim 1$ second. We provide individual estimates with TumE for each of the 150 synthetic tumours, and an additional 750 synthetic tumours of variable sequencing depth from ref ${ }^{16}$, in Supplementary Figures $13 \& 14$. We provide an example TumE output for a synthetic tumour with a single detectable subclone in Figure 2d.

In this study, we note that the birth and death rate were set to fixed values (birth rate $=\log (2)$, death rate $=$ 0 , in line with $\operatorname{ref}^{7}$ ) to additionally improve the computational efficiency of the stochastic simulations of positively selected tumour populations. Therefore, an additional factor that may impact the accurate detection of selection and subclones with TumE is variable birth and death rates in growing tumours. For example, an elevated cell death can lead to an increase in the number of passenger mutations that are swept to higher frequencies during subclonal selection. In regards to the VAF distribution, this elevated number of mutations 'trailing' the subclonal peak may obscure lower frequency subclones or, alternatively, lead to spurious identification of additional subclones due to an elevated number of neutral mutations entering the subclonal frequency range. To assess the impact of variable growth rates, we generated an additional 6 million synthetic tumours across 26 different birth and death rate combinations (simulation parameters outlined in Supplementary Table S1). Overall, we find that our estimates are robust to changes in tumour growth rates. Any errors that do occur only appear to increase the number of parsimonious explanations of the data (e.g., classifying 2 subclones as 1; Supplementary Figure 15). In addition, the prediction of subclone frequency also remained consistent across all the birth and death rate combinations evaluated here (Supplementary Figure 16). 
confound estimates by presenting a spurious number of mutations within subclonal frequency ranges. Although VAFs can be easily corrected for impurity in diploid regions (i.e., diploid VAF / purity), original tumour purity estimates may also be incorrect. To ensure incorrect purity estimates did not confound our predictions, we implemented a peak-finding method that adjusts the VAF distribution to a theoretical $100 \%$ purity state (Methods). Using this adjustment approach, analyses in simulated datasets showed that TumE estimates can maintain a false positive rate for positive selection of less than $\sim 1-5 \%$ in samples with up to $25 \%$ error in purity estimates and at minimum effective sequencing depths (purity * sequencing depth) as low as $\sim 40 x$ (Supplementary Figure $17 \& 18$ ).

\section{Analysis of whole-genome and exome sequenced tumour biopsies} tumour biopsies commonly used to evaluate mixture model based approaches, namely a deep sequenced ( 320x coverage, 90.7\% purity) acute myeloid leukemia (AML) sample from Griffith et al. ${ }^{31}$ and a deep sequenced ( $226 x$ coverage, $71.2 \%$ purity) breast adenocarcinoma sample retrieved from the pan-cancer analysis of whole genomes (PCAWG) ${ }^{11}$ but originally from ref ${ }^{32}$. In both cases, we recovered the correct evolutionary mode, number of subclones, and subclone frequencies (Figure $3 a \& 3 b$ ). In addition, because we provide accurate subclone frequency estimates, we can perform heuristic clustering of the clonal, subclonal, and neutral tail mutations observed in tumour VAF distributions. (Methods). Heuristic clustering uses the theoretical observation that tumour VAF distributions, at minimum, must be composed of a neutral tail, if sufficient sequencing depth is achieved, and a clonal peak, representing mutations acquired prior to tumour initiation or fixed during cancer evolution. Therefore, by first assigning the frequencies of any detected subclones, we can then cluster subclones, the neutral tail, and clonal regions outward from these frequencies using the variance under any preferred sequencing noise model (e.g., binomial variance). We note here that this heuristic approach does not require any additional explicit clustering methods that require model selection and simply uses the expected variance under a given probability distribution. Notably, this heuristic approach facilitates subclonal clustering at almost zero additional computational cost (as observed in the total runtime per sample of $\sim 1$ s).

We next evaluated TumE in whole-exome sequenced (WES) mismatch repair deficient (MMR) gastroesophageal tumours biopsied across multiple spatially distinct regions (collected from von Loga et al. ${ }^{33}$ ). As evolutionary inference requires high-quality genomes, we only included samples that had a mean effective coverage (mean sequencing depth * purity) greater than $60 x$ and a minimum purity of $50 \%$. We note that $\sim 70 \mathrm{x}$ mean sequencing depth has been suggested as the minimal threshold for accurate estimates ${ }^{7,16}$, as we also observed (Supplementary Figures $8 \& 9$ ). Following removal of low-quality biopsies, we retained biopsies from two tumours with one tumour retaining 5 spatially distinct (WES) biopsies. TumE estimates in the 5 spatially distinct biopsies from a single tumour revealed the fixation process of a positively selected 

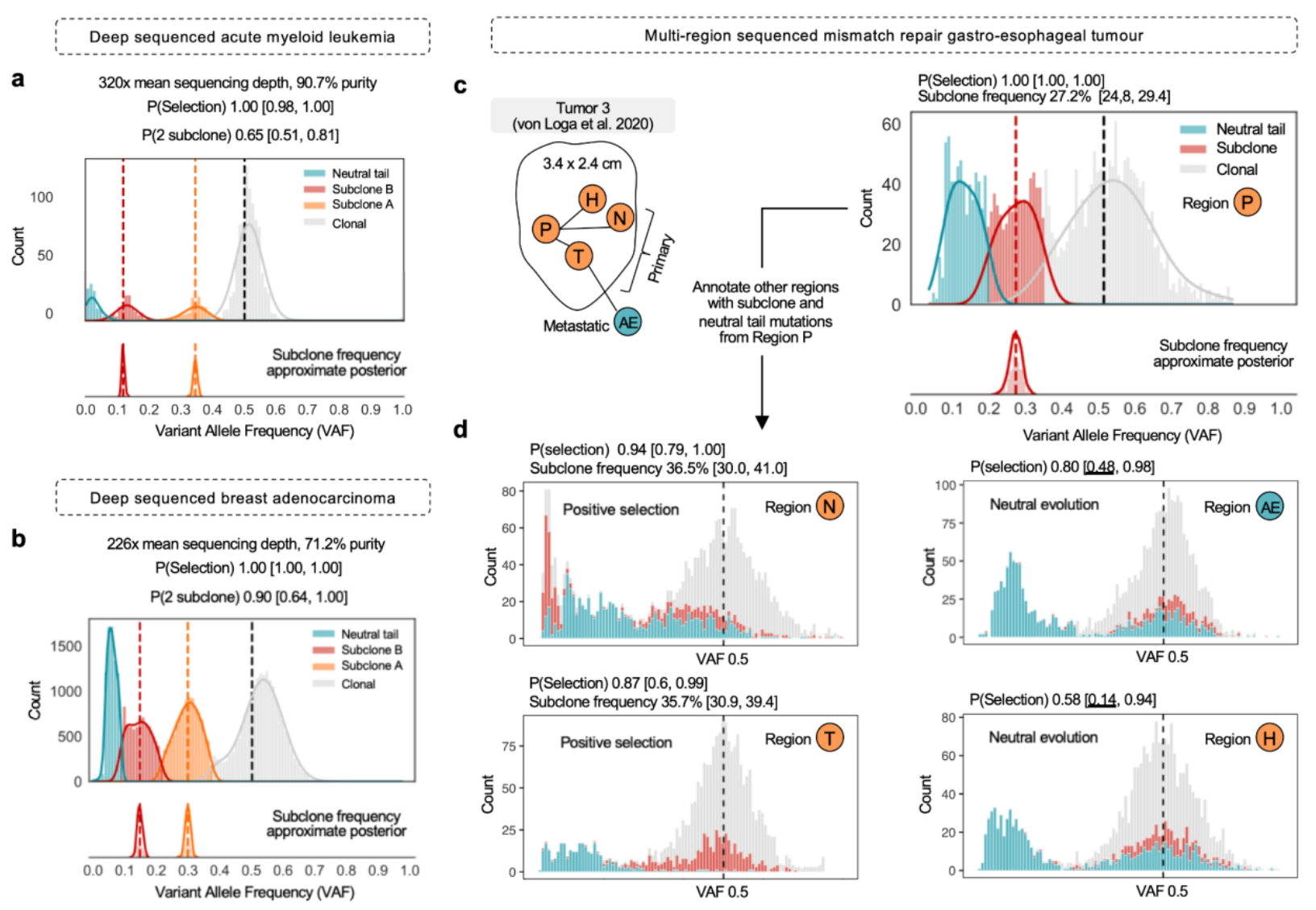

Figure 3. TumE estimates in deep whole-genome or whole exome sequenced tumour biopsies. (a) A deep-sequenced primary acute myeloid leukemia (AML) sample from Griffith et al. ${ }^{31}$. TumE estimated two subclones, a neutral tail, and a clonal peak. $\mathrm{P}(\mathrm{Selection})$ indicates the probability of selection. $\mathrm{P}(0,1,2$ subclone) indicates the probability estimate for the number of subclones. Each probability estimate is provided with the $89 \%$ equal-tailed interval generated from 50 Monte Carlo dropout samples. A sample is labeled positive selection if the lower bound of the $89 \%$ interval is above $\mathrm{P}=0.5$, and the number of subclones is assigned to a sample if the lower bound of the $89 \%$ interval is greater than 0.5 (Methods). Subclone frequency estimates are shown with the complete approximate posterior. (b) A deep-sequenced breast adenocarcinoma from the pan-cancer analysis of whole genomes ${ }^{11}$ (PCAWG). TumE estimated two subclones, a neutral tail, and a clonal peak. (c) We applied TumE to a single mismatch repair deficient (MMR) gastro-esophageal tumour sequenced across 5 spatially distinct regions. We first identified an intermediate frequency subclone in region $\mathrm{P}$ with TumE. (d) Under the hypothesis that TumE could reveal the fixation process of region $\mathrm{P}$ subclones in other regions, we annotated each of the remaining regions with the clonal, subclonal, and neutral tail mutations identified in region $P$. We identified ongoing subclonal selection in 2 out of the 4 remaining regions ( $\mathrm{N}$ and $\mathrm{T}$ ) consistent with an increase in frequency of subclonal and neutral tail mutations from region $\mathrm{P}$. In cases where neutral evolution was the most parsimonious explanation, we observed complete fixation of the region $\mathrm{P}$ subclonal mutations (region $\mathrm{AE}$ and $\mathrm{H}$ ).

Finally, we evaluated TumE in 78 whole-genome sequenced (WGS) tumour biopsies with $>60 x$ mean effective sequencing depth, spanning 10 different cancer types, retrieved from the pan-cancer analysis of whole genomes (PCAWG). To ensure valid inference with TumE, we only used SNVs from high-quality consensus diploid regions and performed purity correction and adjustment (Methods). In total, TumE identified evidence for positive, or subclonal, selection in $50 \%$ of samples whereas the other $50 \%$ were 
adequately described by neutral evolutionary dynamics (Figure 4a \& Supplementary Table S2). Analysis of diploid point mutations in samples with at least one detected subclone $(n=39)$ revealed that $26 \%$ of samples carried a nonsynonymous, indel, or splicing mutation in a known driver gene. The observation that a minor fraction detected subclones harbor point mutations in known drivers is not unexpected as previous work on timing driver mutations has suggested the majority of driver point mutations occur early in tumor development ${ }^{34}$. In this regard, the elevation in subclone frequency may also be driven by a plethora of alternative genomic alterations, such as structural variation, not analyzed here. VAF distributions annotated with TumE fits and driver mutations can be found in Supplementary Figure 19.

Next, to facilitate direct comparison against existing methods, we also applied MOBSTER and sciClone to the 78 WGS samples. Consistent with analyses in simulated tumours, MOBSTER tended to more parsimonious explanations of the data ( $4 \%$ selection, Supplementary Table 3 ) while sciClone (corrected for presence of a neutral tail) estimated excess selection (67\% selection, Supplementary Table 4) relative to TumE (Figure 4a \& 4b). Overall, the general agreement between accurate classification in simulated tumours and estimates in empirical tumours suggested TumE was likely detecting true cases of ongoing selection. When compared to MOBSTER in both simulated and empirical data, the additional subclones detected by TumE can likely be explained by the ability to detect a wider range of subclone frequencies, particularly higher frequency, (Figure 4c) that evade detection by mixture models with flexible beta distributions and model selection strategies that penalize overlapping clusters ${ }^{16}$ (Figure 4c). The identification of additional higher and lower frequency subclones is also consistent with previous theoretical work showing that subclones have a higher probability of being at more extreme or 'peripheral' frequencies in growing tumours ${ }^{14}$.

Previously, alternative methods applied to large cancer cohorts, including PCAWG, have estimated that as few as $3 \%{ }^{16}$ to upwards of $95 \%{ }^{35}$ of samples show evidence for ongoing subclonal selection. The discrepancy is likely explained in modeling approaches and sample selection strategies. For example, in terms of modeling approaches, low estimates are a by-product of utilizing mixture models and model selection strategies that rely on non-overlapping and 'clean' subclonal peaks whereas high estimates likely occur from not considering the neutral dynamics in tumour evolution. In contrast, TumE generates a nonlinear encoding of the VAF distribution, extracting novel representations that increase accuracy while simultaneously accounting for the correct neutrality evolutionary dynamics observed in tumour populations. learning approach. 


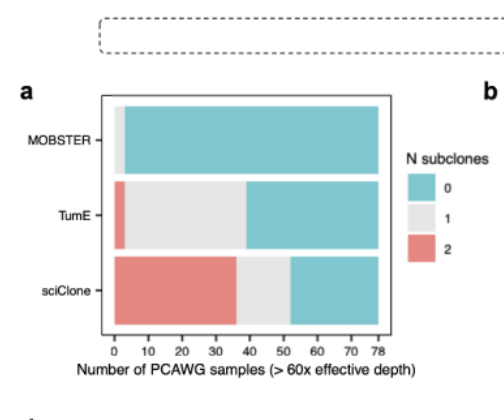

d

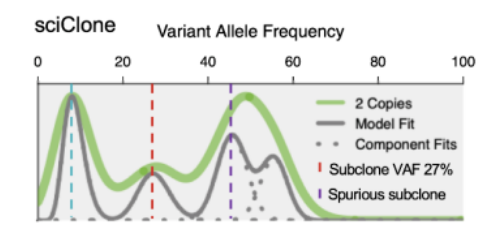

MOBSTER
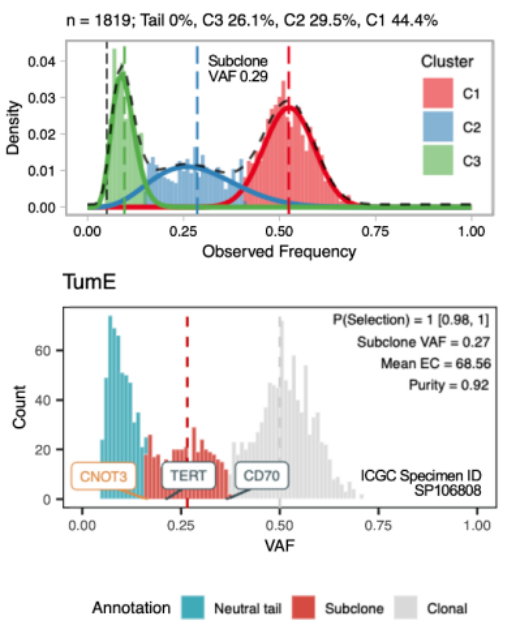

Diploid SNV Driver $\bullet$ Up/Downstream $\bullet$ UTR

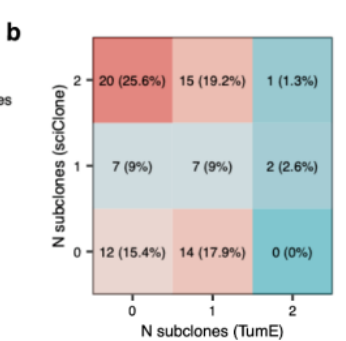

e

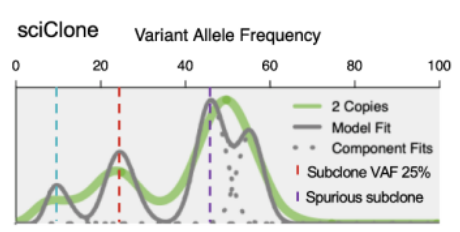

\section{MOBSTER}
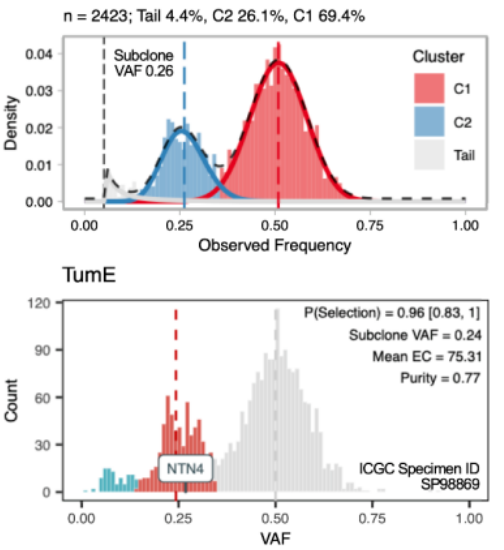

Annotation $\square$ Neutral tail $\square$ Subclone $\square$ Clonal

Diploid SNV Driver $\bullet$ Up/Downstream
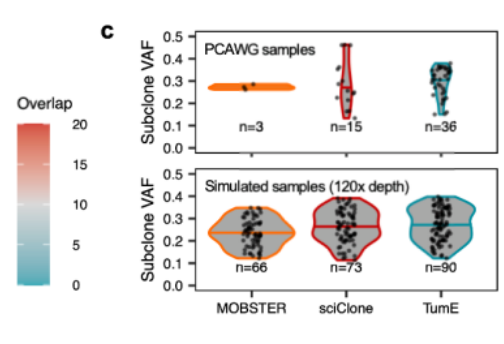

f
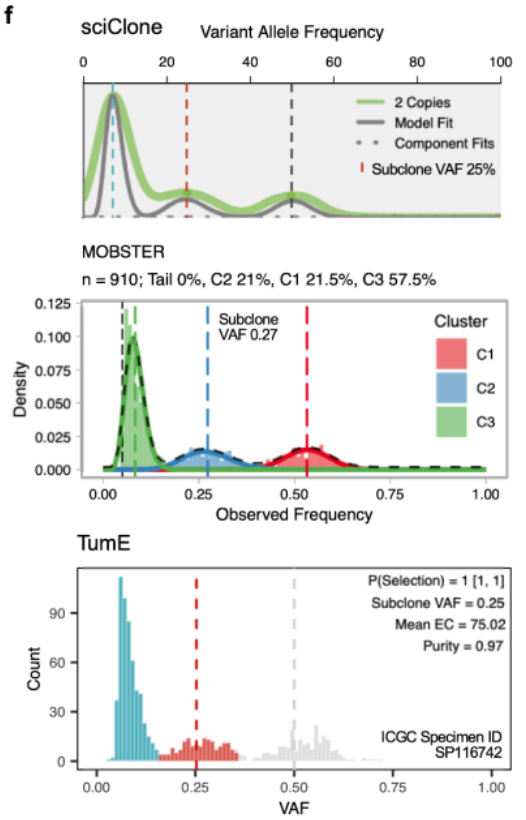

Annotation Neutral tail $\square$ Subclone

Figure 4. Comparison of subclone detection methods in 78 PCAWG samples with mean effective sequencing depth (purity * depth) of greater than 60x. (a) Number of subclone estimates using different methods including MOBSTER, sciClone, and TumE. (b) Comparing agreement and disagreement between existing methods in the analyzed PCAWG samples. (c) Comparison of the estimated subclone frequency in PCAWG samples versus simulated data across different methods. Consistent with simulated data where each method properly identified the presence of 1 subclone, TumE consistently captures higher frequency subclones when compared to MOBSTER. (d - f) Visualization of the 3 samples where each TumE and MOBSTER ${ }^{16}$ detected 1 subclone. sciClone fits are provided for comparison. Single-nucleotide variants (SNV) with annotations in known driver genes ${ }^{8,16}$ were labeled if present.

In addition, the discrepancy in percentage of samples subject to positive selection across recent studies may also be a by-product of cohort selection and sample filtering. We note that in this study we only included PCAWG samples with $>60 x$ mean effective sequencing depth as it represented thresholds for accurate inference of selection by TumE. This differs from previous work by Caravagna et al. 2020 (MOBSTER) where samples were selected based on a post-clustering metric, namely the presence of a neutral tail that carried a relatively large proportion of mutations $(>10 \%)$. However, because the majority of the PCAWG samples with $>10 \%$ neutral tail are not sequenced deep enough (effective depth $<60-70 x$ ) to mitigate overdispersion, detecting selection in these samples is either prevented or confounded. We believe selecting samples based on data quality required for detecting selection (e.g., effective depth), rather than post-hoc filtering based on fits, is likely the better strategy for properly quantifying the occurrence of ongoing 
selection. This disagreement in rates of positive selection was also highlighted in this study as only 1 sample was consistently called subclonal across TumE, MOBSTER and sciClone. We present the 3 overlapping samples between MOBSTER and TumE in Figure 4d-f. Overall, our extensive analyses in simulated and empirical tumours suggests that methods considering the neutral evolutionary dynamics, such as TumE and MOBSTER, should be preferred over consistently incorrect methods such as sciClone. However, mixture models using beta distributions and conservative model selection strategies that penalize overlapping clusters (such as MOBSTER) may not be sufficient to capture the full range of subclone frequencies and may represent the baseline rate of ongoing selection in growing tumours.

\section{A transfer learning framework to infer additional evolutionary parameters}

313 One drawback of simulation-based deep learning approaches is the requirement for the repeated generation of synthetic data for training. Although this allows for fast inference at test time through amortization, altering the model's assumptions or changing the parameters being inferred generally requires simulating a completely new set of data and training an entirely new set of models - a computationally expensive process. Practically, overcoming this limitation would provide substantial reductions in the amount of time and data needed to build accurate models and would make simulationbased approaches more accessible to the general user. Therefore, we hypothesized that our trained deep learning models could be used as a source of 'stored' knowledge for related evolutionary inference tasks that also used the VAF distribution as input.

To explore this possibility, we implemented a transfer learning pipeline, based on domain adaptation ${ }^{36,37}$, to make inferences on additional parameters using a previously developed cancer evolution simulator, TEMULATOR $^{38}$, that was built under a similar but modified set of assumptions relative to our multiplicative fitness framework (Methods, viable parameter combinations for detectable subclones outlined in Supplementary Figure 20). In this study, we employ open set domain adaptation ${ }^{37}$ where the structure of the input space, i.e., the VAF distribution, is retained whereas the outputs, the evolutionary tasks, are modified. Briefly, this pipeline involved generating new synthetic tumour sequencing data using TEMULATOR, performing architecture 'renovation' on pre-trained TumE neural networks to replace existing task-specific branches with new ones, and re-tuning the neural network weights and hyperparameters for optimization on the new evolutionary inference tasks (Figure 5a). The evolutionary inference tasks included predicting subclone fitness, subclone emergence time, mutation rate, and subclone cellular fraction (subclone $\mathrm{VAF}^{*}$ 2). To highlight the benefit of using pre-trained models on related evolutionary inference tasks, we opted to update network weights with only 500,000 synthetic VAF distributions, representing only a fraction ( 1.25\%) of the data used in the original training of TumE. Each VAF distribution was generated by simulating synthetic tumours with TEMULATOR at a birth rate of 1 , death rate of 0.2 , final population 
bioRxiv preprint doi: https://doi.org/10.1101/2021.11.22.469566; this version posted January 31, 2022. The copyright holder for this preprint (which was not certified by peer review) is the author/funder. All rights reserved. No reuse allowed without permission. were uniformly sampled from empirically plausible ranges (Supplementary Table S5).

a
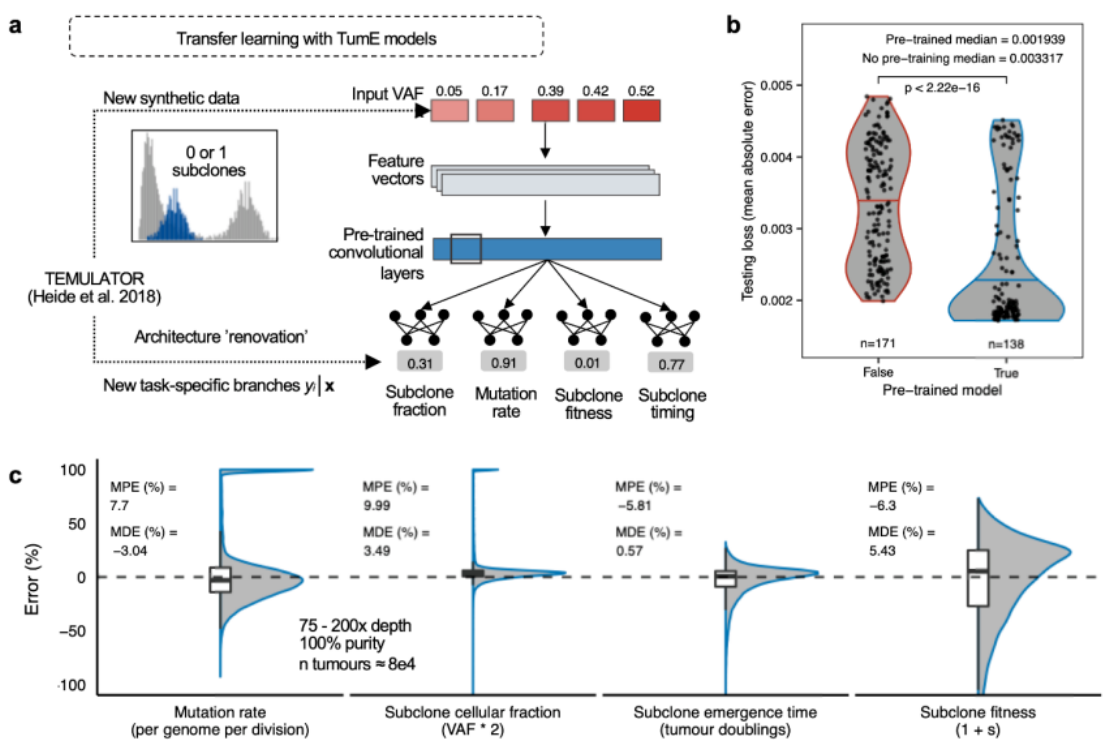

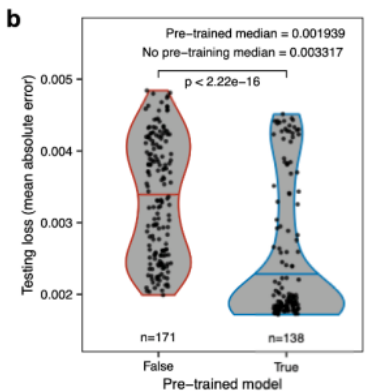

Cancer tissue of origin 自 CLL 追 Liver Pancreas 自 Pancreatic 帛 Prostate
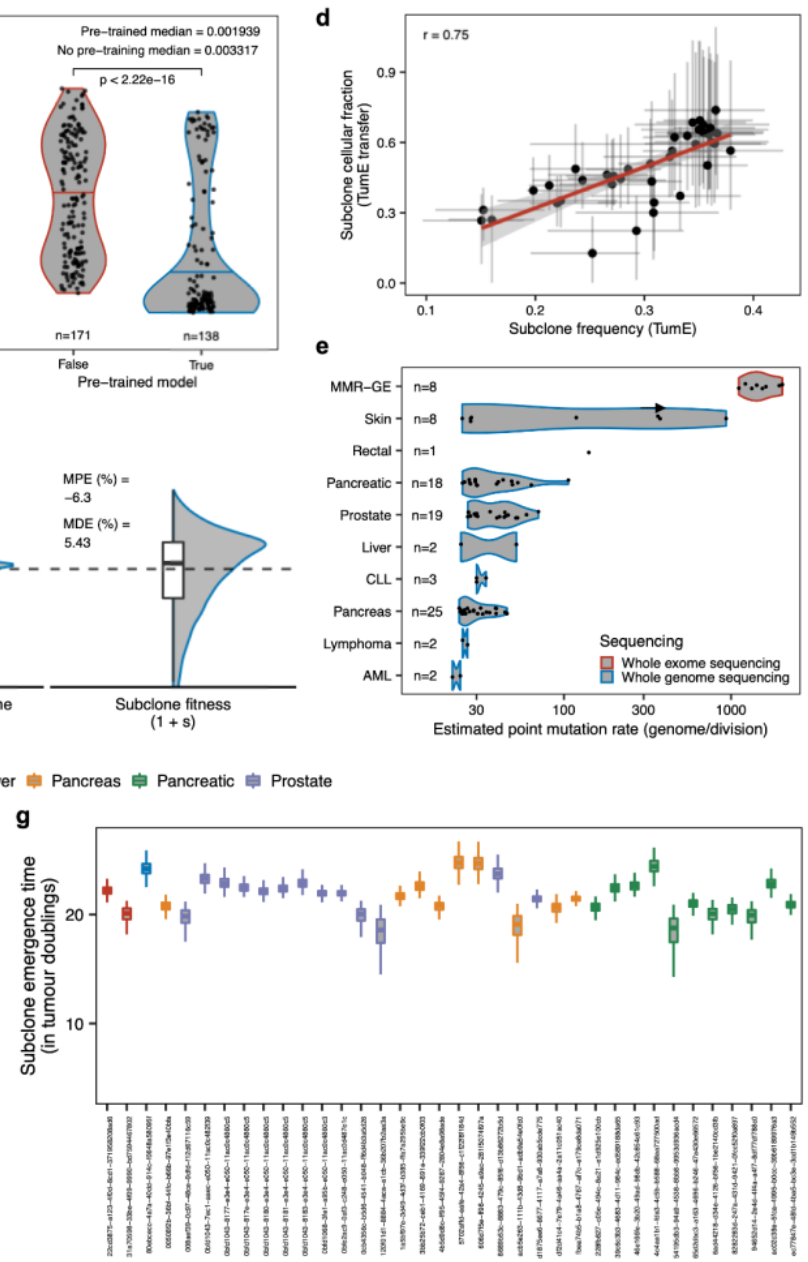

Figure 5. (a) Transfer learning approach utilizing 'renovated' pre-trained neural networks for alternative evolutionary inference tasks in tumour cellular populations. TEMULATOR is an alternative cancer evolution simulator that generates synthetic tumour sequencing data by deterministically initiating subclones at user specified fitnesses and time points ${ }^{38}$. (b) Pre-trained models provide significant reductions in testing loss, over non-pretrained models, when updating neural network weights on reduced dataset size of 500,000 synthetic VAF distributions ( $1.25 \%$ of the total dataset size used to originally train TumE). (c) TumE transfer (TumE-T) effectively recovers evolutionary parameters from TEMULATOR simulations (75-200x mean sequencing depth, 100\% tumor purity) with mean and median percentage errors less than $10 \%$ in all cases. A full description of performance across variable sequencing depths, mutation rates, and subclone frequencies is provided in Supplementary Figure 23. (d) We find consistency between the subclone cellular fraction estimated by TumE-T and the subclone frequency (cellular fraction / 2) estimates generated from TumE, indicating nearly identical tasks are easily transferred through pre-training. (e) Per genome per division mutation rate estimates in 88 WES and WGS samples from von Loga et al. ${ }^{33}$ (MMR-GE = mismatch deficient repair gastro-esophageal cancer), Griffith et al. ${ }^{31}$ (AML $=$ acute myeloid leukemia), and PCAWG ${ }^{11}$. (f) Subclone fitness $(1+s)$ estimates (relative growth rate advantage of subclone over background population) and (g) subclone emergence time estimates in 36 tumour biopsies identified with 1 subclone in the PCAWG data. Subclone fitness and emergence time estimates were scaled to a final tumour population size of $10^{10}$ cells, similar to ref ${ }^{7}$. PCAWG sample identifiers are provided on the $x$-axis. 
comparisons, we performed a random hyperparameter search, tuning the learning rate and number of fully connected layers in the new task specific branches, across both the pre-trained and non-pretrained model groups. Both groups shared identical neural network architectures. When initially evaluating 300 pretrained and non-pretrained models on an external test set of 3000 synthetic tumours, we found that pretrained models obtained significantly lower loss (mean absolute error across all tasks, two-sided Wilcoxon test, $p<2.22 \times 10^{-16}$, Figure $5 b$ ). Further, when evaluating the top performing pre-trained and non-pretrained models on an additional 400,000 synthetic tumours, pre-trained models obtained significantly lower mean percentage errors, relative to non-pretrained models, for predicting the mutation rate, subclone emergence time, subclone fitness, and subclone frequency (two-sided Wilcoxon test, $p<$ $1.7 \times 10^{-8}$ on all tasks, Supplementary Figure 21 ). We note that although 500,000 samples appeared to reach asymptotic predictive performance (Supplementary Figure 22), we have found that as few as 25,000 synthetic samples can generate a model with comparable predictive performance assuming the hyperparameter search space is large enough (e.g., >100 models; Supplementary Figure 22).

Next, we selected the top performing pretrained model, TumE transfer (TumE-T), for further validation. We initially found a modest yet systematic underestimation of the mutation rate ( $50 \%$ mean percentage error). However, this was easily corrected with a post-hoc adjustment by re-fitting the predicted mutation rate to a set of 1000 synthetic tumours using polynomial regression (degree $=2$ ). Evaluating the updated mutation rate estimates on a holdout set of 100,000 synthetic tumours validated the post-hoc adjustment (Supplementary Figure 23). Overall, we were able to effectively recover all evolutionary parameters in the 100,000 synthetic tumours with mean and median percentage errors lower than $10 \%$ in all cases (Figure $5 \mathrm{c})$. The performance was also consistent across sequencing depths and mutation rates, however, as expected, we could only effectively assign subclonal parameters, such as fitness, at detectable subclone frequencies ( 10 - 40\% VAF; Supplementary Figure 24). predicted subclone cellular fraction and subclone frequency estimated by the original TumE models, suggesting nearly identical tasks are easily transferred to new source-target distributions when using pretrained models (Figure $5 \mathrm{~d}$ ). With respect to mutation rates, estimates were consistent with the general trends observed empirically ${ }^{11,33}$ - with mismatch repair deficient tumours showing extremely high mutation rates (>100 per genome per division) and acute myeloid leukemia showing very few (Figure 5e). For subclone fitness and subclone emergence time estimation, we had to consider the difference between simulated and true population sizes ${ }^{7,19}$. In this regard, we rescaled our estimates to account for a true tumour population size of $10^{10}$, similar to ref $^{7}$. With rescaling, TumE-T subclone fitness estimates, defined as the relative growth advantage of the selected subpopulation over the background population, ranged from $\sim 2.1$ to 3.5 (Figure 5f) while subclone emergence time estimates ranged $\sim 18$ to 25 tumour doublings

378 (Figure $5 \mathrm{~g}$ ) in samples with ongoing subclonal selection. We note that emergence times of $\sim 18$ to 25 tumour 
doublings represent approximately 0.001 to $0.16 \%$ of the final tumour volume, which is consistent with theory and empirical evidence suggesting subclones must arise early during tumour growth to reach detectable frequencies ${ }^{7,14,17,39}$.

\section{Discussion}

384 In this study, we developed a synthetic supervised learning approach, TumE, for cancer evolutionary inference. Overall, the synthetic supervised learning approach, TumE, provides four major advantages. First, by generating synthetic data using models of cancer evolution, we are able to explicitly account for the neutral and non-neutral evolutionary dynamics observed in tumour VAF distributions ${ }^{7,12,16}$, thereby avoiding systematic overestimates in the number of subclones due to misclassifying low frequency neutral 'tails'. Second, by using neural networks that can naturally handle high-dimensional VAF distributions as input, we avoid information loss that comes with compressing data into a single statistic, or distance metric, prior to inference, improving model accuracy across all evolutionary inference tasks considered here. Third, by separating simulation and model training from prediction, via amortized inference, we significantly decrease inference time per sample, reducing time from minutes to seconds relative to existing methods. Finally, we show how we can use open set domain adaptation ${ }^{36,37}$, a form of transfer learning, to recycle our models for alternative evolutionary inference tasks that use VAF distributions as input - drastically reducing the number of synthetic samples and computational time required for further model development. Lastly, in contrast to phylogenetic approaches that use mutations to reconstruct or explain the possible history of divergent clonal events in a tumour biopsy, population-genetic based methods, such as TumE, discriminate detectable subpopulations subject based on explicit evolutionary models such as positive selection or neutral evolution using the VAF distribution ${ }^{40,41}$. Altogether, our library of pre-trained models benefits all researchers building inference machines for cancer evolution prediction, even in a non-deep learning setting. For example, providing fast, evolutionary-informed peak initializations for mixture modelbased methods.

We mention some current limitations. Firstly, as a neural network requires optimization on a finite, static set of data, estimates, without transfer learning, are constrained to a pre-defined search space. In this study we focused on cancer evolution in the context of 2 detectable selected subpopulations captured from frequency information in diploid genomic regions. Although multiple studies have shown it's rare to detect 2, or even 1, subclones ${ }^{7,14,16,17}$ in noisy one-dimensional VAF distributions, it's possible we do not capture extreme cases of selected subclonal heterogeneity. Furthermore, focusing on diploid regions may obscure the detection of ongoing selection if mutations are concentrated in copy number aberrated segments. However, constraining analyses to diploid regions provides a strong baseline for model development, while genome-wide linkage provides biological justification for analyzing diploid regions. We also note that copy number variants miscalled as diploid can lead to overestimation of subclonality by introducing spurious mutations in the subclonal frequency range. However, in this study, we focused on using high-quality 
consensus copy number calls extensively validated in pan-cancer cohorts and utilized in previous work used to parse out the evolutionary history of tumours ${ }^{11,16,34}$. Finally, our model of tumour evolution was structured to reflect the biopsy material available here, namely bulk sequenced single site and time point data. We note that tumour growth over space and time can have profound effects on the detectability of selection $^{40,41}$. In this regard, TumE estimates can still be applied in a localized setting and aggregated globally. Nevertheless, more structured ways of integrating a synthetic supervised learning approach with multi-region data are necessary for maximizing utility. fast and accurate amortized estimates that go beyond the current paradigm of single statistics, mixture models, and approximate Bayesian computation for classifying and quantifying ongoing selection in tumour populations. The integration of generative and simulation-based models of cancer evolution with modern deep learning frameworks facilitates robust and efficient estimates of evolutionary and subclonal dynamics in growing tumour populations. This extensible framework provides future avenues for harnessing progressive computational gain for the benefit of cancer genomics and, as an end goal, the cancer patient.

\section{Synthetic data generation}

We generated synthetic data that encoded the evolutionary dynamics observed in the variant allele frequency (VAF) distribution (namely the neutral tail, subclones, and clonal peaks) using two complementary approaches dependent on the underlying evolutionary mode - one for tumors subject to positive selection and one for tumours evolving neutrally.

For tumours simulated under positive selection, we utilized a well-established framework of cancer evolution that models exponential tumour growth under a stochastic branching process ${ }^{7,12,13,15,19,30}$ and coupled this with a virtual biopsy procedure to account for sequencing noise observed in wholegenome/whole-exome sequenced tumours from real patient tumours. For implementation, we adapted a previous cancer evolution framework developed by Williams et al. ${ }^{7}$ Briefly, this model simulates exponentially growing tumour populations under a stochastic branching process using a rejection-kinetic Monte Carlo (MC) algorithm, where a given cell accrues mutations at some Poisson-distributed per

448 branching process continues by randomly sampling existing cells, weighted by cellular fitness, until a final

449 tumour population size $N$, sufficient to recapitulate the features of empirical VAF distributions, is reached.

450 Following completion of each simulation, a virtual biopsy procedure to account for sequencing noise observed in real patient VAF distributions is implemented. In this sequencing noise model, the observed 
frequency for a given mutation $\left(V A F_{o b s}\right)$ relative to the true underlying frequency $\left(V A F_{\text {true }}\right)$ in a tumour of population size $N$ is given by

where $D$ total indicates the total observed read depth, $R$ indicates the number of observed reads covering the mutation, $V A F_{\text {true }}$ indicates the true population frequency of the mutation, and $\rho$ indicates the overdispersion parameter for the beta-binomial.

In this study, we modify the Williams et $\mathrm{al}^{7}$ framework in two ways. Firstly, we implement a fully stochastic arrival of subclones (driver mutations) rather than deterministically injecting a subclone with a specified fitness at a given time $t$. Secondly, the fitness of a subclone or cell is dictated by the multiplicative fitness of all driver mutations. Therefore, when a driver mutation does occur, based on some probability $p_{d}$, it is assigned a selection coefficient $s>0$ sampled from an exponential distribution which increases the cell's growth rate $(b-d)$ by a factor of $(1+s)$ i.e., the fitness. In the case of multiple driver mutations, the fitness of a given cell increases multiplicatively i.e., $\Pi(1+s)$. Although this random injection of driver mutations is more computationally intensive, it implicitly captures a wider variety of potential frequency distributions without hard coding additional parameter settings - for example, when additional subclones, beyond 1 or 2, are present at undetectable frequencies (e.g., $>40 \%$ or $<10 \%$ ). In this study, we consider up to 2 detectable subclones but allow for up to 3 selected subclones to be present at the time of biopsy (see Simulation Parameter Selection below for more details).

For tumours simulated under neutral evolution, we use a generative sampling process for producing neutral VAF distributions, rather than using the stochastic simulation framework. We implement this sampling process because we use a small $N$ population size approximation to generate VAF distributions in our stochastic simulations (using a small $N$ allows us to increase simulation speed and efficiency, which makes generating millions of synthetic VAF distributions practically feasible). Although using a small $N$ is reasonable since the VAF distribution contains no information on population size ${ }^{7}$ (a final simulated tumour population size $N$ of $10^{3}-10^{4}$ has been shown to be sufficient to recapitulate the properties of empirical VAF distributions ${ }^{7}$ ), neutral stochastic simulations have a higher probability of returning lateoccurring spurious subclones due to chance or, in empirical terms, genetic drift. Given the quality of the synthetic data impacts deep learning model performance, we utilize the fully synthetic generative sampling scheme to avoid misspecified data relative to the expected null model of neutral evolution. 
The neutral generative sampling process we implement is based on the observation that neutrally evolving asexual, non-recombining populations, such as cancer populations, have VAF distributions (excluding clonal mutations) that follow a power-law or Pareto distribution ${ }^{16,29}$. Therefore, a VAF for any mutation $i$ in the neutral tail of a frequency distribution can be realized by sampling

where $a$ is the shape parameter and $m$ the scale parameter for the Pareto distribution. parameters that define the Pareto distribution, we can generate realizations of neutral allele frequency distributions that are well-specified. Previously, Caravagna et al ${ }^{16}$ fit Pareto distributions (and beta distributions) to thousands of patient tumours extracting both shape and scale parameters. We used these Pareto distribution fits from diploid regions of patient tumours with greater than $50 x$ sequencing coverage to build sampling distributions for the shape and scale parameters. We then used these sampling distributions to generate allele frequencies under a Pareto distribution and, in addition, randomly assigned clonal mutations to each neutral synthetic VAF. In practice, as previously noted ${ }^{16}$, the scale parameter can be set to the minimum observed frequency as this is the maximum likelihood estimate for the Pareto distribution.

We note that we added additional noise to synthetic neutral distributions to better account for variability observed in empirical data in two ways. Firstly, for any synthetically generated neutral distribution, we randomly trimmed the low frequency neutral tail at a frequency $f\left(10-30 \%\right.$ VAF) with some probability $P_{\text {trim }}$ $(\leq 0.1)$. We perform this step as many VAF distributions observed in patient biopsies lack the characteristic

512 neutral tail, even at high sequencing depth ${ }^{16}$. By randomly trimming neutral synthetic VAF distributions, we 513 tend to more parsimonious explanations of the data, with respect to positive selection, when assessing 514 incomplete and potentially noisy VAF distributions. Furthermore, by adding sequencing overdispersion 515 following trimming, we naturally capture the observed downsampling of degenerate neutral tails in empirical 516 data. Secondly, we randomly shifted the heterozygous, diploid clonal peak (that should be centered at $50 \%$ $517 \mathrm{VAF}$ ) to between 45 and 50\% VAF. We perform this random perturbation of the clonal peak to avoid 518 overestimating positive selection when patient samples have incorrect tumour purity estimates that may 519 have led to spurious elevation in the number of subclonal mutations. other given the alternate data generation approaches, we built an aggregate simulation framework that generated neutral and positive synthetic tumours in pairs - assigning the neutral VAF distributions with 
parameter-matched sequencing noise and equivalent clonal and non-clonal mutations with respect to the paired positive selection simulation.

The synthetic data generation algorithms are outlined in Supplementary and code is available at

\section{Simulation parameter selection}

532 Each stochastic simulation described above was parametrized by the mutation rate (per genome per division), the probability a mutation was a driver, the mean for the exponential selection coefficient distribution, the number of clonal mutations in the founder cell, the maximum number of driver mutation events, the final tumour population size, the sequencing depth, and the sequencing overdispersion. We chose simulation parameters that were consistent with previous studies $7,13,16,30$ and that captured the expected qualitative and quantitative attributes of VAF distributions observed empirically (Supplementary Table S1). All non-fixed parameters were uniformly random sampled during the development of the synthetic datasets. To improve computational speed and efficiency in our stochastic simulations, we used similar simulation approximations as ref $^{7}$. Namely, a small $N$ population size approximation (where we simulated data using a final population size of $10^{3}$ ) and a fixed growth rate (where the birth rate was set to $\log (2)$ and the death rate was set to 0$)$. In addition, as we were focused on differentiating between neutral evolution and selection at effective sequencing depths of $\sim 50-250 \mathrm{x}$, we constrained our search space to 1 or 2 detectable subclones present between $10-40 \%$ VAF. We implemented this constraint as (i) it is extremely rare to detect 3 subclones in a one-dimensional VAF distribution as each subclone has to be $>5$ $10 \%$ VAF (10-20\% cellular fraction) for detection, (ii) most frequency-based methods or studies show limited evidence for detecting $>1$ subclone at 50 - 250x coverage in a single time point, one-dimensional VAF distribution ${ }^{16}$, and (iii) below greater than roughly $10 \%$ VAF subclones merge with the neutral tail and above roughly $40 \%$ VAF subclones begin to merge with the clonal peak when considering diploid regions.

\section{Synthetic supervised learning}

552 As outlined in the results, synthetic or simulation-based deep learning has been shown to be equivalent to 553 amortized approximate inference under a generative model ${ }^{28}$. Therefore, by optimizing a neural network 554 using synthetic VAF distributions sampled from a stochastic generative process $p(\mathbf{x}, \mathbf{z} \mid \boldsymbol{\theta})$ (i.e. the synthetic data generation scheme defined above), we can build fast approximate inference models for evolutionary inference. We describe the synthetic supervised workflow from feature generation to prediction below.

558 Input representation. For each simulation, we converted mutation frequencies into a VAF distribution 559 (histogram) of length $k$ that had a fixed range between $2 \%$ and $50 \%$ VAF. To implicitly condition our model 
above a frequency cutoff based on the variance of a binomial sequencing noise model. We note that this conditioning step is arbitrary and simply acts to improve model optimization during training. In general, a simple approach to conditioning a neural network on a measurable variable involves finding a reasonable encoding within the feature representation. For example, an alternative approach instead of using a frequency cutoff would be to concatenate the sequencing depth to the input feature vector. Overall, each input feature vector was created by counting mutations into $k$ bins where each bin had a width $w$ of (50 $2 \% \mathrm{VAF}) / k$ and a lower frequency cutoff defined by $f_{\text {alt }}+\left(2 \sqrt{f_{\text {alt }} c\left[1-f_{\text {alt }}\right]}\right) / c$ where $f_{\text {alt }}$ is the minimum alternative reads to call a mutation divided by mean sequencing depth and $c$ is mean sequencing depth. For all model development and training, we generated and concatenated two feature vectors with $k=64$ and $k=128$ for each simulation to capture varying levels of information depending on the sparsity of mutations in each synthetic tumour.

Model search. We initially developed neural networks for three single or multi-task inference problems: (i) evolutionary mode (neutral evolution or positive selection) and number of subclones classification ( $\left.M_{m s}\right)$, (ii) frequency prediction for a single subclone $\left(M_{1 s}\right)$, and (iii) frequency predictions for two subclones $\left(M_{2 s}\right)$. For each multi-task, we performed a random hyperparameter search using a one dimensional (1D) convolutional neural network (CNN) with task-specific fully connected branches as a base architecture. For the random search, the hyperparameters included the number of convolutional layers $(1-20)$ the taskspecific branch type (fully connected or global average pooling), the number of feature maps/channels for each convolutional layer (4 - 32), the convolutional kernel width for the left trunk, right trunk, and taskspecific branches $\left(1-17\right.$, odd), the learning rate $\left(10^{-7}-10^{-3}\right)$, and the patience for early stopping ( 3 - 5). To tend toward higher precision and lower recall for predicting selection, we also tuned a penalty term on the positive class in the binary cross entropy loss. Batch size was fixed to 256. Hardswish activations were used at each hidden layer. Dropout, fixed at a probability of 0.5 , was added after each layer to allow for downstream application of uncertainty estimation (see Uncertainty Estimation below). We note that we also explored inferring subclone emergence time under a multiplicative fitness model but could not effectively recover parameters likely due to a complex non-linear relationship between subclonal fitness and emergence time. However, we provide these estimates as an 'experimental' output in the TumE python package (links below).

Model training. We trained over 150 models for each evolutionary inference task(s) using an Adam optimizer, minimizing the cross-entropy loss for classification tasks $\left(M_{m s}\right)$ and the L1 loss for regression tasks ( $M_{1 s}$ and $M_{2 s}$ ), on approximately 40 million synthetic tumours simulated with parameters outlined in

594 Supplementary Table S1. For training, each batch consisted of 20,000 unique simulations and training was 595 stopped after 4 epochs or when early stopping, updated after each batch, was activated based on specified patience. To avoid overrepresentation of any subclone frequency during training, we re-balanced positive 
decimal places (e.g., 0.11 or $11 \%$ VAF). For two subclone simulations, we re-balanced simulations based on the distance between subclones $\left(f_{\text {subclone2 }}-f_{\text {subclone } 1}\right)$ and only included simulations where the distance between subclones was $>5 \%$ VAF. Note that only positive selection simulations were used to train $M_{1 s}$ and M2s.

Model selection. Using an independent test set of one hundred thousand simulations, we then selected the top models across each multi-task for further validation. For $M_{m s}$, we selected models that maximized the mean accuracy across the evolutionary mode, $P$ (Selection), and number of subclones, $P$ ( $N$ subclones), classification, and favoured models that assigned a larger penalty term to misprediction of positive selection (i.e. a lower weight to the positive class in the binary cross entropy loss). For the regression models $M_{1 s}$ and $M_{2 s}$, we selected models that minimized the mean absolute error between the true and predicted subclone frequency on the test set while also ensuring that predictions properly extrapolated across the entire simulated parameter range (e.g., $10-40 \% \mathrm{VAF}$ for subclone frequencies).

Uncertainty estimation. To capture model-based uncertainty in our estimates, we implemented a form of Bayesian approximation for deep learning called Monte Carlo (MC) dropout ${ }^{26,27}$. Conceptually, MC dropout captures model-based uncertainty by taking advantage of the relationship between model averaging and standard dropout - a network with dropout at every layer encodes $2^{n}$ possible network configurations. By keeping dropout on at test time, each prediction is a stochastic pass through a set of randomly activated neurons. More specifically and with a slight abuse of notation w.r.t to ref $^{26}$ ignoring the variational notation, we make estimates of our target variable $y$ (e.g. subclone frequency) by performing $T$ stochastic forward passes through the network and averaging, $E(y)$, the results: where $\hat{y}$ is the output with respect to the input data $x$ for a neural network with $L$ layers, and $W$ corresponds to a weight matrix for each layer $L$. For every stochastic pass, each $W$ is assigned a randomly sampled vector of Bernoulli random variables such that each individual neuron is inactivated with a probability equal to the dropout rate. Under this framework, MC sampling over $T$ stochastic passes through the network generates an approximate posterior for our target variables with respect to the input data. predicting the number of subclones, $\mathrm{P}(N$ subclones $)$, in both synthetic and real patient tumours, we took a conservative, more parsimonious approach to prediction by considering the variance in the approximate

632 posterior. For $\mathrm{P}$ (Selection), we only called positive selection if the lower bound of an $89 \%$ equal-tailed 633 interval for the approximate posterior, computed across 50 stochastic passes through $M_{m s}$, was greater 
than 0.5 . If the lower bound was less than 0.5 , we called neutrality and zero subclones, independent of the result of $\mathrm{P}(N$ subclones $)$. We show the utility of this strategy for mitigating model overconfidence in a synthetic toy example (Supplementary Figure 6). For the regression tasks of predicting subclone frequency and emergence time, we estimated the true value by performing 50 stochastic passes through the networks and averaging the results, while also providing the complete approximate posterior. We describe additional considerations for making estimates in real patient tumour biopsies below.

All model development and training were done using pytorch v1.8.1. We provide a python package, scripts,

642 and all trained neural network models for downloading, use, and modification at

643 https://github.com/tomouellette/TumE.

\section{Model performance in synthetic tumour sequencing datasets}

646 We simulated or collected 3 different datasets of synthetic tumour sequencing data to study the 647 performance of TumE under changing parameter regimes or changes to model assumptions. The first 648 dataset, generated by our simulation framework described above, consisted of $\sim 2.8$ million synthetic 649 tumours simulated across varying sequencing depths and overdispersions (all parameters provided in 650 Supplementary Table S1). Using this dataset, we compared TumE against six frequency-based summary statistics for differentiating between positive selection and neutral evolution. Four of the statistics were cancer evolution statistics developed previously ${ }^{12}$ and provided in the R package neutralitytestr. For each sample, the parameters of neutralitytestr were set as follows: ploidy $=2$, cellularity $=1$, read_depth $=$ simulated mean sequencing depth, rho = simulated overdispersion (rho). Two of the statistics were common population genetic statistics, Tajima's $D^{20}$ and Fay and Wu's $H^{21}$. Only variant allele frequencies and sequencing depth were required for input to compute these statistics. We provide an implementation of Tajima's $D$ and Fay and Wu's $H$ for tumour sequencing data in the github repository. We additionally evaluated a mixture model-based approach MOBSTER $^{16}$ for subclone detection and frequency quantification. To enable analysis of $\sim 2.8$ million synthetic tumours, we ran MOBSTER with the following parameters: $K=1: 3$, samples $=2$, init $=$ "peaks", tail $=c(T R U E, F A L S E)$, epsilon $=1 e-6$, maxlter $=100$, fit.type $=$ "MM", seed $=12345$, model.selection $=$ "relCL", pi_cutoff $=0.02$, N_cutoff $=10$. We defined the number of subclones that MOBSTER detected as follows. If a tail and 3 beta components were fit then we assigned 2 subclones, if a tail and 2 beta components or if no tail and 3 beta components were fit we assigned 1 subclone, and for all remaining fits we assigned 0 subclones or neutrality.

The second dataset was retrieved from Caravagna et al. ${ }^{16}$ and consists of synthetic tumour sequencing data from 150 tumours sequenced to $120 x$ depth using a beta-binomial sequencing model and grown to a size of $>10^{8}$ at a birth rate of 1 and death rate of 0.2 . The complete description is provided in the

669 supplementary of ref $^{16}$. We used this dataset to evaluate the small $\mathrm{N}$ approximation and to compare TumE 670 to existing mixture model methods. We applied both MOBSTER and a variational Bayesian mixture model 671 sciClone ${ }^{24}$ to this dataset. MOBSTER was run under default package settings without parallel computation 
672 and with $\mathrm{K}=1$ to 3 beta components. sciClone was run under default package settings with copyNumberCalls fixed to 2 and maximumClusters fixed to 4 . To estimate the number of selected subclones with sciClone, which doesn't account for neutral evolutionary dynamics, we took the inferred number of

675 subclones and subtracted 2 (representing the neutral tail and clonal peak). Per-sample runtimes for TumE,

676 MOBSTER, and sciClone were computed on a single machine with 16GB memory and a 2.3GHz quadcore Intel i7 processor.

The third dataset was used to evaluate variable birth and death rates on TumE estimates for predicting positive selection, determining the number of subclones, and estimating subclone frequency. The dataset consisted of $\sim 6$ million synthetic tumours, generated by our simulation framework described above, grown at variable birth and death rate combinations. Mutation rate and mean sequencing depth were both fixed to 100. Other parameters were uniformly sampled and all parameters evaluated are outlined in Supplementary Table S1.

The fourth dataset was used to evaluate the robustness of TumE estimates to variation in tumour purity. We simulated 6000 synthetic tumours at sequencing depths of 20 - 200x with a beta-binomial overdispersion of rho $=0.03$ and tumour purities ranging from $20-100 \%$. We then made an auxiliary dataset the impact of incorrect purity estimates on TumE predictions. To generate this dataset, we added -25 to $25 \%$ error on each purity estimate. In total, 10 datasets with varying error on purity estimates were generated. We then performed purity correction and adjustment of the VAFs from each synthetic tumour prior to evaluating the false positive rate for positive selection with TumE. A more detailed outline of the purity correction and adjustment strategy is outlined below.

\section{Evolutionary parameter estimates in bulk sequenced single tumour biopsies}

697 In this study, we used diploid regions of patient tumours for evolutionary inference as we did not have access to accurate phased mutation information for copy number correction of VAFs at non-diploid sites. However, in the absence of complete whole-genome duplication, mutated diploid regions should be sufficient to capture ongoing selection, due to selective sweeps from genome-wide linkage, if a sufficient number of neutral passengers mutations are accumulated during cell division over time ${ }^{7,16}$. We note that, similar to ref ${ }^{16,34,35}$, we only used high-quality diploid consensus calls (star 3 ) from the original PCAWG study to avoid potential biases from miscalled segments appearing as subclones in the VAF distribution.

704 Samples with fewer than 100 mutations following removal of low-quality regions were not included in this 705 study. 
have shown that tumour genomes sequenced below 50-70x coverage are exceedingly noisy and have insufficient limits of detection relative to low-frequency mutations for proper evolutionary inference ${ }^{16,31}$. impurity, where purity (cellularity) is defined as the percentage of cells in the biopsy that are of malignant or tumour origin. In general, low tumour purity can lead to spurious identification of subclones as it results in lower observed VAFs relative to the true underlying population VAFs. To ensure our inferences weren't biased by impurity, we corrected all VAFs using corresponding tumour purity estimates collected from the study of origin where $\mathrm{VAF}_{\text {corrected }}=\mathrm{VAF}_{\text {observed }} /$ purity.

We also note that some purity estimates may be incorrect - in these cases updating the VAFs with incorrect purity estimates can lead to a heterozygous clonal cluster (that should be centered at approximately $50 \%$ $\mathrm{VAF}$ ) in the subclonal frequency range ( 10 - 40\% VAF). To ensure clonal clusters were properly centered at $50 \%$ VAF following purity correction, we performed additional adjustments to each patients' VAF distribution using the following heuristic. We first computed the density for each VAF distribution and then identified all the locations where the second derivative of the density was zero i.e., peak finding. If the closest peak to 50\% VAF (the theoretical diploid clonal cluster) was above 35\% VAF, we considered it a misrepresented clonal peak. We made this assumption as analyses in pan-cancer datasets suggest that all tumours are initiated in somatic cells already carrying mutations ${ }^{10,11}$. We then fit a Gaussian distribution to the identified clonal cluster of each patient VAF distribution and adjusted each VAF by multiplying by 0.5 divided by the mean of the fit. Although a Beta distribution is generally used for fitting clonal clusters in cancer genomics, a Gaussian is a reasonable approximation for adjusting VAFs based on incorrect purity estimates as it provides accurate estimates of the cluster mean, and has been used in previous subclonal clustering methods ${ }^{42}$. The quality of this adjustment strategy (false positive rate for positive selection) was evaluated in 6000 synthetic tumours simulated across sequencing depths of $20-200 x$, tumour purities of $20-100 \%$, and purity estimate errors ranging from -25 to $25 \%$ (Supplementary Figures 17 \& 18).

Heuristic clustering using the estimated subclone frequencies was performed either using the expected variance under a binomial sequencing noise model or, alternatively, using the subclone frequency estimates to initialize the means of a gaussian mixture model. Clustering under the binomial framework was performed as follows. Given an estimated subclone frequency $q$, all mutations within the frequency range of $q \pm$ $(\varepsilon \sqrt{q c[1-q]}) / c$ were assigned to the subclone, where $\varepsilon$ scales the cluster width and $c$ is the mean

743 For an empirical comparative analysis against existing methods, we fit both MOBSTER and sciClone to the

74478 PCAWG samples. For MOBSTER, we used default settings that enabled fitting up to 3 beta components 745 with a tail using an 'ICL' model selection strategy. We also attempted to use the 'relCL' model selection 
strategy; however, we were generating excessive positive selection calls relative to the original study and therefore opted for the ICL strategy. For sciClone, we used default settings as specified in the github repository (https://github.com/genome/sciclone). We report both ICL and reICL fits for MOBSTER in Supplementary Table S3 and sciClone fits in Supplementary Table S4.

\section{Transfer learning for inference in alternative synthetic data regimes} output space $Y_{S}$, transfer learning attempts to use pre-training to improve the performance on another target domain $T$ composed of $X_{T}$ and $Y_{T}$. We employ a variant of transfer learning called open set domain adaptation ${ }^{37}$ to take advantage of our pre-trained models for additional inference tasks. In this case, the input spaces remain constant (Xs $=X \mathrm{Xt}$, VAF distribution) but the inferred tasks are allowed to differ. Open set indicates that some tasks may overlap with the output space of both the source and target domains.

To provide a concrete use case for transfer learning in synthetic supervised learning, we aimed to infer additional evolutionary parameters such as subclone fitness, subclone emergence time, mutation rate, and subclone cellular fraction (subclone frequency * 2 ) using synthetic tumour sequencing data generated by an alternative cancer simulation framework TEMULATOR ${ }^{38}$. TEMULATOR differs from our synthetic data generation scheme, which was built around a multiplicative fitness driver model, as subclones are deterministically initiated at user specified emergence times and fitnesses. To facilitate transfer between previous and new tasks, we performed architecture renovation on the pre-trained neural networks, retaining all convolutional layers while replacing existing task-specific fully-connected branches with new taskspecific fully connected branches ( 4 in total). To maximize the amount of information transferred to new tasks, we combined the convolutional layers from both the $M_{m s}$ and $M_{1 s}$ models described above.

We then simulated 500,000 synthetic tumours at a birth rate of 1 , death rate of 0.2 , and final population size of $10^{4}$ (additional parameters such as mutation rate were uniformly sampled and are outlined in Supplementary Table S3). To facilitate efficient simulation, we first fit a noisy Gaussian process (GP) regression to the viable emergence time and fitness parameter combinations (that generated detectable subclones between $\sim 10-40 \%$ VAF) and used the GP to sample viable emergence times given a uniformly sampled fitness. We made the GP noisy to facilitate parameter combinations that resulted in subclones across the entire frequency range. The GP was fit using three kernels (RBF with length scale 100, dotproduct, and white noise) and an alpha of $10^{-6}$ in the python package scikit-learn v1.0. Next, we used the simulations to re-optimize the pre-trained model weights, using an Adam optimizer to minimize the L1 loss (mean absolute error) for predicting new evolutionary inference tasks. To ensure fair benchmarking between networks with and without pre-trained weights, we performed a random hyperparameter search with 150 pre-trained and 150 non-pretrained models, tuning the learning rate and number of fully 
was generated under similar parameter settings. We corrected modest yet systematic overestimates in mutation rate ( $\sim 50 \%$ mean percentage error) in the final transferred model by fitting a polynomial (degree 2) ridge regression in scikit-learn $v 1.0$ to the predicted mutation rates. The mutation rate adjustment was performed using VAF distributions from 1000 synthetic tumours. We validated the correction on an additional 100,000 synthetic tumours. All TEMULATOR synthetic tumours were generated using parameter settings in Supplementary Table S3.

Predictions in empirical samples were performed by taking 500 Monte Carlo dropout samples and averaging the results. Dropout was only activated at test time on the new task-specific branches. Per-base mutation rate estimates in whole-exome sequenced MMR-GE samples were rescaled based on the 60MB Agilent SureSelectXT Human All Exon v6 kit used in the original study ${ }^{33}$. Because subclone fitness and emergence time is impacted by final tumour size, we rescaled our estimates to a realistic tumour size of $10^{10}$ cells, similar to ref $^{7}$. Previous work ${ }^{7}$ has shown that given subclone frequency $f_{\text {sub }}$ and an estimated final population size $N_{\text {end }}$, the age of a tumour at time of biopsy can be estimated by $t_{\text {end }}=\log 2\left(\left[1-f_{\text {sub }}\right] *\right.$ $\left.N_{\text {end }}\right)$. Therefore, given that the relationship between emergence time in tumour doublings and log population size is linear, we can generate a rescale fitness estimate $w_{R}$ as follows.

$$
w_{R}=1+(w-1) * \frac{t_{\text {end }}-t_{s}}{t_{\text {end }}-t_{s_{R}}}
$$

where $w$ equals subclone fitness, tend indicates time at tumour biopsy or final population size in tumour doublings, and $t_{s}$ indicates the time of subclone emergence in tumour doublings. $R$ subscript indicates values rescaled to population size of $10^{10}$. The parameters $f_{s u b}, w$, and $t_{s}$ are all inferred. We approximate the rescaled subclone emergence time $t_{s_{R}}$ as $t_{s} * \log \left(N_{\text {end }}\right) / \log \left(N_{\text {end }}\right)$.

\section{Data availability}

808 All TumE predictions in synthetic and empirical datasets, intermediate processing data, data used for generating figures, and fully trained deep learning models can be found at the Zenodo reposityory https://doi.org/10.5281/zenodo.5931436. Whole-genome sequenced AML samples were retrieved from Griffith et al ${ }^{31}$. Multi-region whole-exome sequenced mismatch deficient repair gastro-esophageal samples were retrieved from von Loga et $\mathrm{al}^{33}$. The remaining whole-genome sequenced samples were retrieved from $P C A W G^{11}$. We provide hosting of the electronic supplementary at https://tomouellette.gitlab.io/ouellette awadalla 2021/.

\section{Code availability}

Scripts for generating figures and analyses can be found at https://doi.org/10.5281/zenodo.5931436. Code for generating synthetic tumour sequencing data can be found at 
819 https://github.com/tomouellette/CanEvolve.jl. Code for performing inference with TumE can be found at 820 https://github.com/tomouellette/TumE.

821

\section{References}

1. Hanahan, D. \& Weinberg, R. A. Hallmarks of Cancer: The Next Generation. Cell 144, 646-674 (2011).

2. Tabassum, D. P. \& Polyak, K. Tumorigenesis: it takes a village. Nat. Rev. Cancer 15, 473-483 (2015).

3. Lipinski, K. A. et al. Cancer Evolution and the Limits of Predictability in Precision Cancer Medicine. Trends Cancer 2, 49-63 (2016).

4. Minussi, D. C. et al. Breast tumours maintain a reservoir of subclonal diversity during expansion. Nature 1-7 (2021) doi:10.1038/s41586-021-03357-x.

5. Fittall, M. W. \& Loo, P. V. Translating insights into tumor evolution to clinical practice: promises and challenges. Genome Med. 11, 1-14 (2019).

6. West, J. B. et al. Multidrug Cancer Therapy in Metastatic Castrate-Resistant Prostate Cancer: An Evolution-Based Strategy. Clin. Cancer Res. 25, 4413-4421 (2019).

7. Williams, M. J. et al. Quantification of subclonal selection in cancer from bulk sequencing data. Nat. Genet. 50, 895-903 (2018).

8. Martincorena, I. et al. Universal Patterns of Selection in Cancer and Somatic Tissues. Cell 171, 1029-1041.e21 (2017).

9. Gatenby, R. A. \& Brown, J. S. Integrating evolutionary dynamics into cancer therapy. Nat. Rev. Clin. Oncol. 17, 675-686 (2020).

10. Weinstein, J. N. et al. The Cancer Genome Atlas Pan-Cancer analysis project. Nat. Genet. 45, 1113-1120 (2013).

11. Campbell, P. J. et al. Pan-cancer analysis of whole genomes. Nature 578, 82-93 (2020).

12. Williams, M. J., Werner, B., Barnes, C. P., Graham, T. A. \& Sottoriva, A. Identification of neutral tumor evolution across cancer types. Nat. Genet. 48, 238-244 (2016).

13. Sun, R. et al. Between-region genetic divergence reflects the mode and tempo of tumor evolution. Nat. Genet. 49, 1015-1024 (2017).

14. Bozic, I., Paterson, C. \& Waclaw, B. On measuring selection in cancer from subclonal mutation frequencies. PLOS Comput. Biol. 15, e1007368 (2019).

15. Lee, N. \& Bozic, I. Inferring parameters of cancer evolution from sequencing and clinical data. bioRxiv 2020.11.18.387837 (2020) doi:10.1101/2020.11.18.387837. 
16. Caravagna, G. et al. Subclonal reconstruction of tumors by using machine learning and population genetics. Nat. Genet. 52, 898-907 (2020).

17. Tung, H.-R. \& Durrett, R. Signatures of neutral evolution in exponentially growing tumors: A theoretical perspective. PLOS Comput. Biol. 17, e1008701 (2021).

18. Crow, J. F. \& Kimura, M. An introduction to population genetics theory. (New York, Evanston and London: Harper \& Row, Publishers, 1970).

19. Salichos, L., Meyerson, W., Warrell, J. \& Gerstein, M. Estimating growth patterns and driver effects in tumor evolution from individual samples. Nat. Commun. 11, 732 (2020).

20. Tajima, F. Statistical Method for Testing the Neutral Mutation Hypothesis by DNA Polymorphism. Genetics 123, 585-595 (1989).

21. Fay, J. C. \& Wu, C. I. Hitchhiking under positive Darwinian selection. Genetics 155, 1405-1413 (2000).

22. Sheehan, S. \& Song, Y. S. Deep Learning for Population Genetic Inference. PLOS Comput. Biol. 12, e1004845 (2016).

23. Prangle, D. Summary Statistics in Approximate Bayesian Computation. ArXiv151205633 Math Stat (2015).

24. Miller, C. A. et al. SciClone: Inferring Clonal Architecture and Tracking the Spatial and Temporal Patterns of Tumor Evolution. PLOS Comput. Biol. 10, e1003665 (2014).

25. Roth, A. et al. PyClone: statistical inference of clonal population structure in cancer. Nat. Methods 11, 396-398 (2014).

26. Gal, Y. \& Ghahramani, Z. Dropout as a Bayesian Approximation: Representing Model Uncertainty in Deep Learning. ArXiv150602142 Cs Stat (2016).

27. Gal, Y. \& Ghahramani, Z. Bayesian Convolutional Neural Networks with Bernoulli Approximate Variational Inference. ArXiv150602158 Cs Stat (2016).

28. Le, T. A., Baydin, A. G., Zinkov, R. \& Wood, F. Using Synthetic Data to Train Neural Networks is Model-Based Reasoning. ArXiv170300868 Cs Stat (2017).

29. Kessler, D. A. \& Levine, H. Large population solution of the stochastic Luria-Delbrück evolution model. Proc. Natl. Acad. Sci. 110, 11682-11687 (2013).

30. McFarland, C. D., Mirny, L. A. \& Korolev, K. S. Tug-of-war between driver and passenger mutations in cancer and other adaptive processes. Proc. Natl. Acad. Sci. 111, 15138-15143 (2014).

31. Griffith, M. et al. Optimizing Cancer Genome Sequencing and Analysis. Cell Syst. 1, 210-223 (2015).

32. Nik-Zainal, S. et al. The Life History of 21 Breast Cancers. Cell 149, 994-1007 (2012). 
33. von Loga, K. et al. Extreme intratumour heterogeneity and driver evolution in mismatch repair deficient gastro-oesophageal cancer. Nat. Commun. 11, 139 (2020).

34. Gerstung, M. et al. The evolutionary history of 2,658 cancers. Nature 578, 122-128 (2020).

35. Dentro, S. C. et al. Characterizing genetic intra-tumor heterogeneity across 2,658 human cancer genomes. Cell 184, 2239-2254.e39 (2021).

36. Kouw, W. M. \& Loog, M. An introduction to domain adaptation and transfer learning. ArXiv181211806 Cs Stat (2019).

37. Busto, P. P. \& Gall, J. Open Set Domain Adaptation. in 2017 IEEE International Conference on Computer Vision (ICCV) 754-763 (IEEE, 2017). doi:10.1109/ICCV.2017.88.

38. Heide, T. et al. Reply to 'Neutral tumor evolution?' Nat. Genet. 50, 1633-1637 (2018).

39. Sottoriva, A. et al. A Big Bang model of human colorectal tumor growth. Nat. Genet. 47, 209216 (2015).

40. Schwartz, R. \& Schäffer, A. A. The evolution of tumour phylogenetics: principles and practice. Nat. Rev. Genet. 18, 213-229 (2017).

41. El-Kebir, M. SPhyR: tumor phylogeny estimation from single-cell sequencing data under loss and error. Bioinformatics 34, i671-i679 (2018).

42. Chkhaidze, K. et al. Spatially constrained tumour growth affects the patterns of clonal selection and neutral drift in cancer genomic data. PLOS Comput. Biol. 15, e1007243 (2019).

43. West, J., Schenck, R. O., Gatenbee, C., Robertson-Tessi, M. \& Anderson, A. R. A. Normal tissue architecture determines the evolutionary course of cancer. Nat. Commun. 12, 2060 (2021).

44. Xiao, Y. et al. FastClone is a probabilistic tool for deconvoluting tumor heterogeneity in bulksequencing samples. Nat. Commun. 11, 4469 (2020). 